\title{
Therapeutic Drug Monitoring of Rivastigmine and Donepezil Under Consideration of CYP2D6 Genotype-Dependent Metabolism of Donepezil
}

This article was published in the following Dove Press journal: Drug Design, Development and Therapy

\author{
Marion Ortner' \\ Marion Stange \\ Heike Schneider' \\ Charlotte Schröder ${ }^{2}$ \\ Katharina Buerger ${ }^{3}$ \\ Claudia Müller ${ }^{3}$ \\ Felix Müller-Sarnowski ${ }^{1}$ \\ Janine Diehl-Schmid (1D' \\ Hans Förstl' \\ Timo Grimmer ${ }^{1, *}$ \\ Werner Steimer ${ }^{2, *}$ \\ 'Department of Psychiatry and \\ Psychotherapy, Technical University of \\ Munich, School of Medicine, Klinikum \\ rechts der Isar, Munich, Germany; \\ ${ }^{2}$ Institute for Clinical Chemistry and \\ Pathobiochemistry, Technical University \\ of Munich, School of Medicine, Klinikum \\ rechts der Isar, Munich, Germany; \\ ${ }^{3}$ Institute for Stroke and Dementia \\ Research (ISD), University of Munich, \\ School of Medicine, Klinikum der \\ Universität München, Munich, Germany \\ *These authors contributed equally to \\ this work
}

Background: The efficacy of acetylcholinesterase inhibitors (AChE-I) might depend on blood concentration. While rivastigmine metabolism is independent of the cytochrome P450 system, its isoenzymes, especially CYP2D6, metabolize donepezil. CYP2D6 polymorphisms can cause altered enzyme activity resulting in lower or higher than expected drug concentrations of donepezil.

Objective: We investigated correlations between clinical efficacy and serum concentrations of rivastigmine and donepezil under special consideration of CYP2D6 genotype or gene dose-dependent metabolism of donepezil.

Methods: Serum concentrations of donepezil and rivastigmine were measured by liquid chromatography - tandem mass spectrometry (LC-MS/MS). Real-time quantitative polymerase chain reaction (PCR) and allele-specific PCR were performed to assess CYP2D6 genotype and gene dose.

Results: Patients treated with rivastigmine $(n=28)$ or donepezil $(n=48)$ were included in the study. Both gene dose and metabolism type significantly predicted the level of donepezil serum concentration ( $p=0.019$ and $p=0.013$, respectively). In the rivastigmine group, changes of the word list delayed recall subtest before treatment and under stable medication were significantly associated with rivastigmine serum levels $(\beta=0.465 ; p=0.018)$. Drug serum concentrations were outside the recommended range in a substantial percentage of participants, which might have contributed to poor correlations between changes in cognitive measures and drug concentrations. Donepezil serum concentrations significantly depended on CYP2D6 gene dose.

Conclusion: Testing AChE-I serum concentration should be considered in patients without clinical response to treatment or those with severe side effects. Patients with donepezil drug levels outside the recommended range might additionally profit from CYP2D6 genotyping or treatment with an AChE-I independent of CYP metabolism.

Keywords: Alzheimer's disease, cytochrome P-450 enzymes, genetic polymorphism, therapeutic drug monitoring, mass spectrometry

\section{Introduction}

Acetylcholinesterase inhibitors (AChE-I) such as donepezil, galantamine, and rivastigmine are recommended for the treatment of mild to moderate Alzheimer's dementia. $^{1-5}$ One factor that may influence the efficacy of AChE-I is blood drug concentration. However, studies investigating this association have shown inconsistent results. $^{6-14}$ Blood concentration, in turn, might depend on the way the substance is metabolized. Whereas rivastigmine is mainly hydrolyzed and the inactive metabolite
Correspondence: Marion Ortner Email marion.ortner@tum.de 
renally excreted, ${ }^{15}$ donepezil is metabolized by CYP 450 isoenzymes 2D6 and 3A4 in the liver and undergoes glucuronidation. CYP2D6 polymorphisms can result in altered metabolism rates. ${ }^{16-19}$ Even though CYP3A4 is majorly involved in donepezil metabolism, genetic polymorphisms do not seem to relevantly influence donepezil clearance and clinical outcome. ${ }^{20,21}$ For this reason, we are going to focus on CYP2D6 polymorphisms in this study.

Depending on CYP2D6 activity, the corresponding phenotypes can be classified as poor metabolizers (PM) with no enzyme activity, intermediate metabolizers (IM) with reduced enzyme activity, extensive metabolizers (EM) with normal enzyme activity, and ultra-rapid metabolizers (UM) with increased enzyme activity. Approximately $15 \%$ of patients with Alzheimer's disease (AD) might be classified as either PM or $\mathrm{UM}^{22}$ Instead of correlating the phenotype with the genotype, Steimer et al recommend correlating the phenotype with a semiquantitative gene dose that is dependent on the number and activity of CYP2D6 alleles present. ${ }^{23}$ They further suggest correlating the gene dose and not the genotype with the phenotype.

Even though a large genetic variability of the hepatic metabolism of donepezil has been reported, ${ }^{24}$ neither the CYP2D6 genotype nor donepezil blood concentrations are routinely tested in clinical settings. Metabolism and subsequently concentrations of donepezil are also affected by concomitant treatment with CYP2D6 inhibitors or CYP2D6 inducers. ${ }^{25}$ Other factors influencing donepezil or rivastigmine blood concentrations have been described, such as sex, ${ }^{20}$ age, ${ }^{26-28}$ weight,${ }^{15,25}$ daily dose,${ }^{26}$ and duration of treatment. ${ }^{11}$

Despite high clinical relevance, only a few studies have investigated the association between CYP2D6 polymorphism, donepezil plasma concentrations, and clinical efficacy of donepezil. While most studies did not find a statistically significant correlation between allele frequency and donepezil concentration or clinical outcome, ${ }^{10,11,14,29}$ a few did. ${ }^{28,30}$

So far no single study has compared different AChE-I in terms of correlations between blood concentrations and clinical efficacy.

\section{Objectives}

In this study we investigated two AChE-I - donepezil, that is hepatically metabolized and rivastigmine, which is renally excreted - in regard to correlations between blood concentrations and clinical efficacy while putting a special focus on the role of CYP2D6 genotype and gene dose-dependent metabolism of donepezil.

\section{Methods}

The methods have already been described elsewhere. ${ }^{31}$

\section{Ethics Statement}

The study protocol was approved by the ethics committee of the Faculty of Medicine of the Technical University of Munich, Munich (reference number 673/02). All patients provided written informed consent prior to any studyspecific procedures. All clinical investigations have been conducted in accordance with the principles of the Declaration of Helsinki, sixth revision.

\section{Patient Recruitment and Study Design}

The study was conducted at the outpatient unit of the Centre for Cognitive Disorders at the Department of Psychiatry, Klinikum rechts der Isar, Technical University of Munich, Munich, Germany and the Memory Clinic at the Institute for Stroke and Dementia Research, Klinikum der Universitaet Muenchen, Munich, Germany between October 2012 and December 2014. Patients had initially been referred for the diagnostic evaluation of cognitive impairments by selfreferral, general practitioners, neurologists, psychiatrists, or other institutions, and had undergone a standardized diagnostic procedure that has been described previously. ${ }^{32}$ It included an interview with the patient and an informant, obtaining demographic data, medical history, concomitant medication, physical, neurological, and psychiatric examinations, a neuropsychological evaluation including the Mini-Mental State Examination (MMSE), ${ }^{33}$ the Consortium to Establish a Registry for Alzheimer's Disease Neuropsychological Assessment Battery (CERAD-NAB) ${ }^{34}$ and the clock drawing test, ${ }^{35}$ as well as a routine laboratory screening test. The treating physician started patients diagnosed with mild to moderate Alzheimer's dementia ${ }^{36}$ on treatment with an AChE-I by his own choice in accordance with the German treatment guidelines for Alzheimer's dementia. ${ }^{2}$ Mild dementia was defined by a MMSE of 26-20 points and moderate by 19-10 points. German treatment guidelines recommend AChE-I, such as donepezil, rivastigmine, or galantamine, for symptomatic treatment of mild and moderate Alzheimer's dementia. ${ }^{2}$ Each doctor is independent in choosing which AChE-I he prescribes. Besides medical reasons, such as known skin conditions and allergies, the patients' preferences for oral or transdermal applications are usually taken into account.

At a routine follow-up appointment after 3-6 months, patients were informed about the study and asked to provide written informed consent if they met the inclusion criteria. 
Patients needed to be able to provide written informed consent and have started treatment with either donepezil capsules or rivastigmine transdermal patch after the initial assessment. They should be compliant in taking their medication and have reached steady state. The only study-specific procedure consisted of a venous blood draw in order to assess serum concentrations of donepezil and rivastigmine and the genotype and gene dose of CYP2D6 alleles. Routine follow-up procedures included a patient and caregiver interview with an assessment of the current medication, the start of AChE-I treatment, the time point when the dose of that AChE-I was increased, side effects of AChE-I treatment, a neuropsychological assessment (CERAD-NAB, MMSE, clock drawing), and a psychiatric and a neurological examination.

Patients were excluded from study participation if they were incapable to provide written informed consent, were not started on or not on a stable dosage of donepezil or rivastigmine, or if dementia was due to any other disease than Alzheimer's disease, for instance, Parkinson's disease. Further exclusion criteria were other possible causes of cognitive impairment such as sedating psychotropic medication (eg, tricyclic antidepressants, low-potent antipsychotics), substance misuse, clinical signs of major depression, or major abnormalities in routine blood testing at the initial presentation of the patient.

For the correlation between donepezil serum concentration and gene dose an à-priori power analysis was calculated: using 158 patients' correlations of $\rho=0.8$ will be detected with a $97.5 \%$ confidence interval.

\section{Blood Sample Collection and Analyses}

We aimed to measure steady-state trough drug levels. To achieve this, the patient needed to be on the respective AChE-I at least 5 half-times and blood was drawn as close as possible to the time the next dose would have been due. If the blood draw could not be achieved prior to the scheduled time point, medication was held until after the blood draw.

Serum concentrations of donepezil and rivastigmine were measured by liquid chromatography - tandem mass spectrometry (LC-MS/MS). Standard procedures were used for genotyping. Depending on allele status subjects were classified into PM, IM, EM, and UM (Supplementary Table 1). Additionally, as proposed by Steimer et $\mathrm{al}^{23}$ an individual gene dose was assigned to each allele to calculate a semiquantitative gene dose (Supplementary Tables 1-3). Details of blood sample collection, LC-MS/MS method and genotyping and gene dose assignment are provided in Supplementary Materials.

\section{Cognitive Assessments and Evaluation of Treatment Efficacy}

Efficacy of the treatment with donepezil or rivastigmine was evaluated by changes in the results of cognitive assessment tests before initiation of an AChE-I and under stable treatment. The absolute differences in points scored in the initial and follow-up assessment for the individual subtests, as well as the sum score for the CERAD-NAB were calculated. Negative values indicated worsening, positive values indicated improvement of cognitive functions.

\section{Statistical Analyses}

Subjects under treatment with donepezil or rivastigmine were characterized using descriptive statistics. All statistics were calculated using SPSS Statistics 23 (SPSS Inc., Chicago, IL, USA). A significance threshold of 0.05 (twosided) was applied. Group comparisons for normally distributed variables were calculated using $t$-test, for not normally distributed variables, Mann-Whitney $U$-test was applied.

In the donepezil group only, we assessed the correlation between serum concentration of donepezil and gene dose of CYP2D6 using linear regression analysis. The dependent variable was donepezil serum concentration; the independent variable of interest was gene dose. The analysis was controlled for other independent variables chosen based on their known influence on donepezil concentrations. These variables consisted of the daily dose of donepezil, concomitant medication with CYP2D6 inhibitors (citalopram and carvedilol each as separate factor), age, sex, duration of treatment with donepezil, and time since last dosing. As a confirmative analysis, the same model was calculated using metabolism type instead of gene dose.

Since serum concentrations also depend on tissue mass, body weight and body mass index (BMI) were included as independent variables in the respective regression model. BMI was calculated by the formula body weight $[\mathrm{kg}] /$ height $[\mathrm{m}]^{2}$.

The differences between scores in the subtests of the CERAD-NAB of the initial assessment and the follow-up assessment were calculated to investigate correlations between serum concentration and efficacy of the respective treatment. In a second step, linear regression analyses with the differences in the individual subtest as the dependent variable were calculated. The independent variables were serum concentration, results of CERAD-NAB subtest at initial assessment, time between initial assessment and follow-up, sex, and age.

We decided on the CERAD-NAB wordlist delayed recall subtest as the primary analysis (i) to avoid multiple 
comparisons and (ii) because we expected most variability in this test and it is the best discriminatory measure in the CERAD-NAB to distinguish between healthy controls and subjects with even mild dementia. ${ }^{37}$ The regression analyses using the other CERAD-NAB subtests were considered exploratory. Accordingly, uncorrected p-values are reported and a value of $p<0.05$ was considered statistically significant.

All statistical models were tested for interaction. There was no imputation of missing data.

\section{Results}

\section{Patients' Characteristics}

Characteristics of participants are shown in Table 1. There were no statistically significant differences between the 48 subjects in the donepezil group and the 28 subjects in the rivastigmine group regarding demographic data, time-tofollow-up, duration of treatment with donepezil or rivastigmine, body weight, BMI (Table 1), or test results of the initial neuropsychological assessment (Supplementary Table 4). Six subjects in the donepezil group were treated with weak CYP2D6 inhibitors (Table 1). Supplementary Figure 1 shows a flow diagram of the progress of participants.

\section{Serum Drug Concentration and Gene Dose \\ Drug Serum Concentrations}

The next AChE-I dose was not administered until after the blood draw, which occurred $1.25 \pm 2.4(-10$ to +4$)$ hours before the time point of the next scheduled drug administration. Drug serum concentrations are shown in Table 2. Serum concentration of donepezil $10 \mathrm{mg}$ daily showed a very high variance of $242 \mathrm{ng}^{2} / \mathrm{ml}^{2}$, which differed significantly from the variance of $26.5 \mathrm{ng}^{2} / \mathrm{ml}^{2}$ under treatment with a rivastigmine patch of
$9.5 \mathrm{mg}$ daily $(\mathrm{p}<0.001)$. Variation coefficients of serum measurements did not differ between groups (Table 2). Donepezil serum concentrations were significantly higher in female subjects (all dosages $\mathrm{p}=0.021 ; 10 \mathrm{mg}$ daily $\mathrm{p}=0.004$ ), while body weight was lower $(\mathrm{p}<0.001)$. The BMI did not significantly differ between sexes (Table 3 ). Rivastigmine serum concentrations did not differ between sexes (Supplementary Table 5). Serum concentrations for the donepezil and rivastigmine group are shown for the respective cohort in its entirety and additionally divided by sexes in Figure 1.

\section{Genotype and Gene Dose}

In 47 participants in the donepezil group, CYP2D6 polymorphisms were analyzed. For one subject the analysis could not be completed. Tested alleles, their frequency and gene doses are shown in Supplementary Table 6 . Supplementary Table 7 shows the distribution of gene dose, type of metabolizer and the respective donepezil serum concentration. For Boxplots of the distribution of donepezil serum concentrations for the respective gene doses, please see Supplementary Figure 2.

\section{Associations Between Donepezil Levels and CYP2D6 Gene Dose}

The applied linear regression model was significant $(p<0.001)$ and explained $47.3 \%$ of the variability of serum drug concentration. Gene dose $(\beta=-0.342 ; p=0.019)$, daily donepezil dose $(\beta=0.378 ; p=0.003)$, duration of donepezil treatment $(\beta=0.285 ; \mathrm{p}=0.038)$, and $\operatorname{sex}(\beta=-0.532 ; \mathrm{p}<0.001)$ were significant predictors of drug serum concentration (Table 4 and Figure 2).

In univariate regression analyses no hints for significant interactions were found; forcing interaction terms into the model did not improve the model fit (Supplementary Table 8).

Table I Characteristics of Participants

\begin{tabular}{|l|l|l|l|}
\hline & Donepezil & Rivastigmine & P-value \\
\hline Numbers & 48 & 28 & - \\
Sex: male:female & $23: 25(48 \%: 52 \%)$ & $16: 12(57 \%: 43 \%)$ & $0.435^{\mathrm{a}}$ \\
Age (in years) & $72.6 \pm 8.89(53-88)$ & $72.5 \pm 6.9 I(53-86)$ & $0.960^{\mathrm{b}}$ \\
Time baseline to follow-up (in days) & $216.8 \pm 70.92(80-44 \mathrm{I})$ & $225.5 \pm 78.40(122-442)$ & $0.979^{\mathrm{c}}$ \\
Duration from start of AChE-I treatment to follow-up (in months) & $5.7 \pm 2.53(2-13)$ & $5.59 \pm 2.09(2-12)$ & $0.905^{\mathrm{c}}$ \\
Number of participants taking CYP2D6 inhibitors & $6($ Citalopram x5; Carvedilol xI) & n.a. & - \\
Number of participants taking CYP2D6 inducers & none & n.a. & - \\
Weight (in kg) $\mathrm{n}=42$ (Donepezil) $\mathrm{n}=25$ (Rivastigmine) & $69.5 \pm 9.14(50-83)$ & $68.5 \pm 13.55(47-90)$ & $0.764^{\mathrm{b}}$ \\
BMI (in kg/m $\left.{ }^{2}\right) \mathrm{n}=42$ (Donepezil) $\mathrm{n}=25$ (Rivastigmine) & $23.9 \pm 3.14(I 7.2-34.5)$ & $23.8 \pm 3.77(16.5-30.9)$ & $0.853^{\mathrm{b}}$ \\
\hline
\end{tabular}

Notes: where applicable: mean \pm standard deviation (minimum-maximum); p-values calculated from ${ }^{a}$ Chi-squared test, ${ }^{b} t$-test or ${ }^{\mathrm{C}}$ Mann-Whitney-U-test.

Abbreviations: AChE-I, acetylcholinesterase inhibitor; kg, participants' weight in kilogram; BMI, body mass index. 
Table 2 Serum Concentrations of AChE-I

\begin{tabular}{|c|c|c|c|}
\hline & Donepezil & Rivastigmine & p-value \\
\hline Daily dose (number of participants) & $\begin{array}{l}15 \mathrm{mg}(1) ; 10 \mathrm{mg} \\
(4 \mathrm{I}) ; 5 \mathrm{mg}(6)\end{array}$ & $\begin{array}{l}9.5 \mathrm{mg}(26) \\
4.6 \mathrm{mg}(2)\end{array}$ & \\
\hline Serum concentration in $\mathrm{ng} / \mathrm{mL}$ in all participants & $\begin{array}{l}38.9 \pm 16.50 \\
(12.95-87.55)\end{array}$ & $\begin{array}{l}6.2 \pm 5.10 \\
(0-17.50)\end{array}$ & \\
\hline $\begin{array}{l}\text { Serum concentration in } \mathrm{ng} / \mathrm{mL} \text { in participants under treatment with donepezil } 10 \mathrm{mg} / \mathrm{d} \text { or } \\
\text { rivastigmine } 9.5 \mathrm{mg} / \mathrm{d} \text {, respectively }\end{array}$ & $\begin{array}{l}41.2 \pm 15.56 \\
(18.8-87.6)\end{array}$ & $\begin{array}{l}6.5 \pm 5.14 \\
(0.47-17.50)\end{array}$ & \\
\hline Variance of serum concentration with donepezil $10 \mathrm{mg}$ or rivastigmine $9.5 \mathrm{mg} / \mathrm{d}$, respectively & 242.0 & 26.5 & $<0.00 \mathrm{I}^{\mathrm{a}}$ \\
\hline Variation coefficient of serum concentration measurement & $\begin{array}{l}5.2 \pm 2.44 \\
(0.57-10.32)\end{array}$ & $\begin{array}{l}5.9 \pm 3.75 \\
(1.05-17.4 I)\end{array}$ & $0.775^{\mathrm{b}}$ \\
\hline Time since last dosing (in h) & $\begin{array}{l}22.2 \pm 2.13 \\
(14-26)\end{array}$ & $\begin{array}{l}23.6 \pm 2.95 \\
(15-28)\end{array}$ & $0.001^{b}$ \\
\hline
\end{tabular}

Notes: Daily drug dose, serum concentration, and variance of blood serum concentration for donepezil and rivastigmine group. Where applicable: mean \pm standard deviation (minimum-maximum). ${ }^{\mathrm{a} C}$ Calculated from F-test. ${ }^{\mathrm{b}}$ Calculated from Mann-Whitney U-test.

Abbreviations: AChE-I, acetylcholinesterase inhibitor; mg, milligram; ng, nanogram; mL, milliliter; d, day; h, hours.

Table 3 Sex Differences Donepezil Group: Serum Concentration and BMI of Male and Female Participants

\begin{tabular}{|l|l|l|l|}
\hline & Male & Female & p-value \\
\hline $\begin{array}{l}\text { Serum concentration } \\
\text { donepezil (in } \mathrm{ng} / \mathrm{mL})\end{array}$ & $\begin{array}{l}33.1 \pm 12.0 \\
(12.95-56.65)\end{array}$ & $\begin{array}{l}44.2 \pm 18.44 \\
(14.85-87.55)\end{array}$ & 0.021 \\
\hline $\begin{array}{l}\text { Serum concentration on } \\
\text { daily dose of IOmg }\end{array}$ & $\begin{array}{l}34.8 \pm 11.17 \\
(18.75-56.65)\end{array}$ & $\begin{array}{l}48.0 \pm 16.83 \\
(23.4-87.55)\end{array}$ & 0.004 \\
\hline Body weight (in kg) & $\begin{array}{l}74.7 \pm 5.9 \\
(60-83)\end{array}$ & $\begin{array}{l}64.8 \pm 9.1 \\
(50-83)\end{array}$ & $<0.001$ \\
\hline BMI (in kg/m $\left.{ }^{2}\right)$ & $\begin{array}{l}23.8 \pm 2.33 \\
(17.2-26.5)\end{array}$ & $\begin{array}{l}24.0 \pm 3.79 \\
(18.6-34.5)\end{array}$ & 0.762 \\
\hline
\end{tabular}

Notes: Where applicable: mean \pm standard deviation (minimum-maximum). $\mathrm{p}$-value calculated from Mann-Whitney-U-test

Abbreviations: $\mathrm{ng}$, nanogram; $\mathrm{mL}$, milliliter; $\mathrm{mg}$, milligram; kg, participants' weight in kilogram; BMI, body mass index.

As a confirmative analysis, the same regression model was calculated using metabolism type instead of gene dose which yielded similar results with metabolizer status, daily donepezil dose, concomitant treatment with CYP2D6 inhibitors, and sex as significant predictors for donepezil serum concentration (corrected $\mathrm{R}^{2}=0.482$, $\mathrm{p}<0.001$; Supplementary Table 9).

To rule out spurious correlation caused by differences in body weight between sexes additional linear regression analyses with body weight or BMI as additional independent variables were conducted. Both models were significant $(p<0.001)$ and explained $43.9 \%$ and $43.4 \%$ of variability of serum drug dose, respectively, comparable to the original analysis (Supplementary Tables 10 and 11 ).

\section{Explorative Analysis of Influence Factors on Rivastigmine Drug Serum Concentration}

As a plausibility check, a regression analysis to explore possible predictors of rivastigmine serum concentration was conducted including daily dose of rivastigmine, concomitant medication with CYP2D6 inhibitors, age, sex, duration of treatment with rivastigmine and time since last dosing as independent variables. Neither the regression model $\left(p=0.767\right.$, corr. $\left.R^{2}=-0.112\right)$ nor any of the variables were statistically significant (Supplementary Table 12), even after adding body weight or BMI as independent variables (Supplementary Tables 13 and 14).

\section{Correlations Between AChE-I Serum Concentrations and Changes in the Results of the Neuropsychological Assessments}

Treatment groups did not significantly differ regarding the efficacy of AChE-I treatment based on neuropsychological assessments with marked variability in both groups (Supplementary Table 15).

Results of multivariate regression analyses for individual CERAD-NAB subtests and the sum-score are shown in $\underline{\text { Supplementary Tables } 16}$ and 17. In both treatment groups, the "word list delayed recall" subtest yielded a significant model. However, only the rivastigmine serum drug concentration significantly predicted the variability of this subtest 

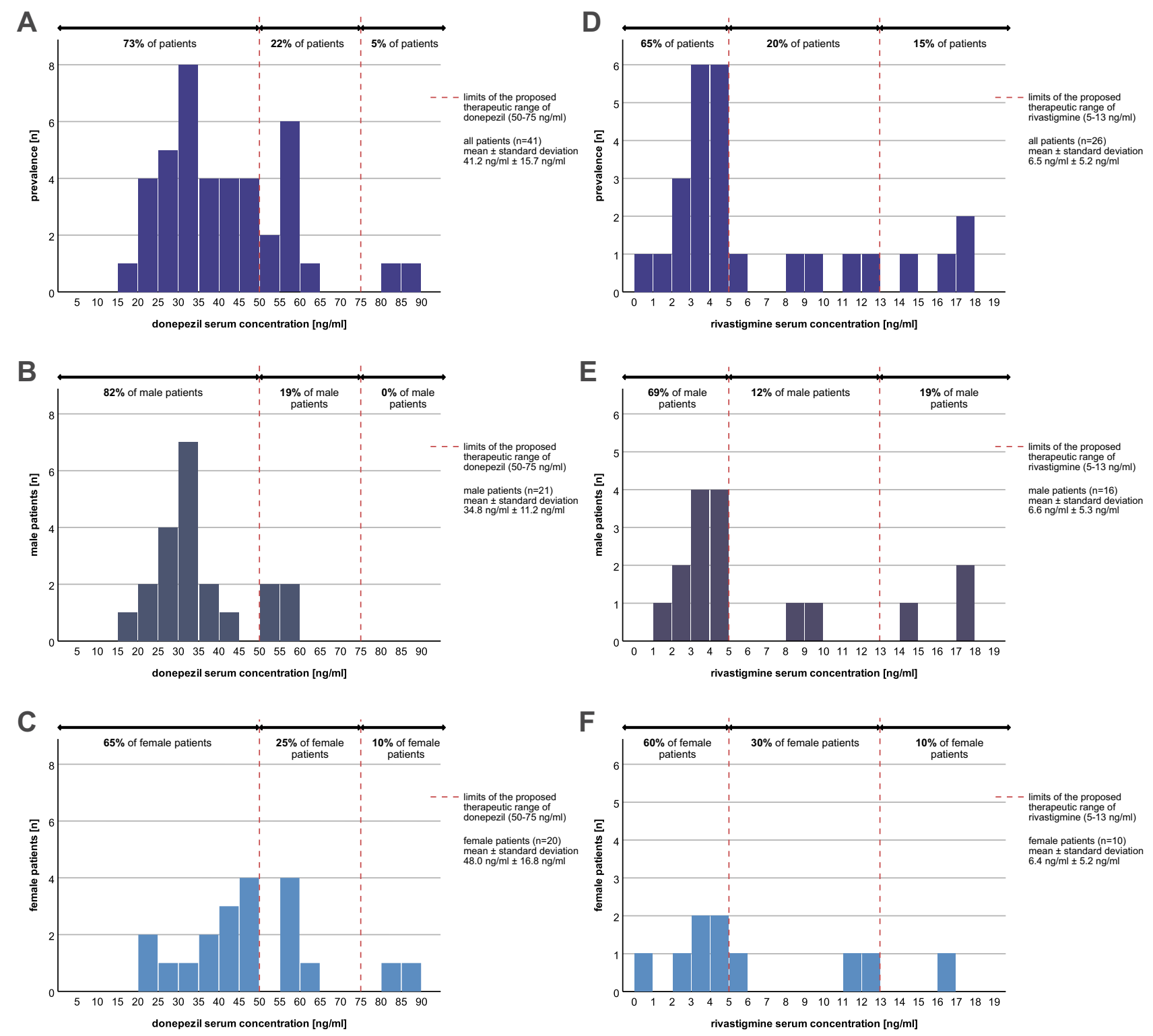

Figure I Participants treated with donepezil $10 \mathrm{mg}$ per day or rivastigmine $9.5 \mathrm{mg}$ per day: distribution of serum concentration in relation to the therapeutic range and sex. Notes: (A) shows donepezil serum concentrations, (D) rivastigmine serum concentrations for the respective sample. As we found a significant difference in donepezil serum concentration between sexes ( $p=0.004$, Table 3 ) and as sex was a significant predictor for donepezil serum concentration ( $<<0.00 \mathrm{I}$, Table 4 ), we additionally depict serum concentrations for each sex (for donepezil (B and $\mathbf{C})$ ), for rivastigmine (E and $\mathbf{F}$ ). The proposed therapeutic range for donepezil (A, B, C) and rivastigmine (D, E, F) according to the AGPN Consensus Guidelines is shown between the red dotted lines. ${ }^{40}$

Abbreviations: $\mathrm{ng} / \mathrm{mL}$, nanograms per milliliter; $\mathrm{n}$, number of participants.

$(\beta=0.465 ; \mathrm{p}=0.018)$ and the regression model explained $26.9 \%$ of the variability (adjusted $\mathrm{R}^{2}=0.269$ ). Results of all univariate models are shown in Supplementary Tables 18 and 19.

\section{Discussion}

We investigated the interrelations between treatment efficacy, serum concentration, and CYP2D6 genotype in $\mathrm{AD}$ patients treated with rivastigmine or donepezil, respectively. Extending previous studies, we not only looked at the metabolism type but also calculated a semiquantitative gene dose for each participant in the donepezil group. Groups did not significantly differ in terms of demographics or results of the assessments. Both gene dose and metabolism type significantly predicted $(\mathrm{p}=0.010$ and $\mathrm{p}=0.007$, respectively) the level of donepezil serum concentration. The subtest word list delayed recall in the rivastigmine group $(\beta=0.465 ; p=0.018)$ was significantly associated with the serum concentration. 
Table 4 Prediction of Serum Concentration of Donepezil: Multivariate Regression Model

\begin{tabular}{|l|l|l|}
\hline & Beta & p-value \\
\hline Gene dose & -0.342 & 0.019 \\
Daily dose & 0.378 & 0.003 \\
CYP2D6-inhibitors & 0.213 & 0.071 \\
Age & 0.160 & 0.165 \\
Sex & -0.532 & $<0.001$ \\
Duration of AChE-I treatment & 0.285 & 0.038 \\
Time since last dosing (in h) & -0.016 & 0.900 \\
\hline
\end{tabular}

Notes: $n=47$; corr. $R^{2}=0.473 ; p<0.001$

Abbreviations: AChE-I, acetylcholinesterase inhibitor; h, hours.

\section{Factors Influencing Donepezil Concentration}

Sex seems to affect donepezil but not rivastigmine concentration. In our study female participants had 35\% higher concentrations ( $\mathrm{p}=0.004)$, a finding that has been previously described. ${ }^{12}$ A possible explanation for this finding could be the relationship between increased donepezil clearance and increased body weight. ${ }^{25}$ According to the drug label information, donepezil clearance increases from $7.77 \mathrm{~L} / \mathrm{h}$ for a body weight of $50 \mathrm{~kg}$ to $14.04 \mathrm{~L} / \mathrm{h}$ for a body weight of $110 \mathrm{~kg}$. Therefore, the higher donepezil serum concentrations we saw in female participants might be partially explained by their significantly lower body weights resulting in a lower donepezil clearance compared to male participants. Not surprisingly, the average body weight of male participants in our study was significantly higher than of female participants, while BMI did not significantly differ between sexes. However, we did not see a statistically significant influence of either body weight or BMI on donepezil concentration. This suggests that there might be other, sex-specific factors influencing pharmacokinetics and thus donepezil serum concentrations.

We were surprised by the significant increase in donepezil concentration depending on the duration of donepezil treatment as all subjects in our study were considered to be in a steady state regarding donepezil metabolism. We do not have a good explanation for the mechanism behind this accumulation of donepezil over time. However, this finding is in line with a study by Miranda et al who also described an increasing donepezil concentration over a 12month treatment duration. ${ }^{11}$

Although drug label information states an age-dependent reduced elimination rate of donepezil ${ }^{25}$ Noetzli et al could not confirm this in their study ${ }^{20}$ and neither did we see a statistical significant influence of patient age on donepezil serum concentration.

\section{Correlation Between Genetics and Donepezil Concentration}

Our analyses confirmed our hypothesis of a correlation between the gene dose or the metabolism type and donepezil serum concentration. Both gene dose and metabolism type predicted significantly $(\mathrm{p}=0.010$ and $\mathrm{p}=0.007$, respectively) the level of donepezil serum concentration.

To our knowledge, this is the first time the gene dose of CYP2D6 as a parameter for the genetically determined metabolism rate was correlated with donepezil serum concentration. There are few previous studies that have investigated CYP2D6 mutations and donepezil blood concentration and results are inconsistent. ${ }^{11,14,29,30,38}$

In our study, in addition to the gene dose and the type of metabolism, the daily dose of donepezil had a significant effect on donepezil serum concentration. This is in line with literature regarding pharmacokinetics of donepezil. ${ }^{39}$

Concomitant medication with CYP2D6 inhibitors might affect long-term blood concentration. This factor was borderline significant in our study $(\mathrm{p}=0.066$ for gene dose; $\mathrm{p}=0.031$ for type of metabolizer). We have no knowledge of studies specifically investigating the direct influence of CYP2D6 inhibitors on donepezil plasma concentration. Comparable to our result, Noetzli et $\mathrm{al}^{20}$ found a significant influence of CYP2D6 inhibitors on the clearance of donepezil which would automatically affect the plasma concentration. In our study, six patients in the donepezil cohort were treated with weak CYP2D6 inhibitors (citalopram or carvedilol) that are not thought to be of clinical relevance (Table 1). As a plausibility analysis, we post hoc calculated a new linear regression analysis without these patients. The analysis yielded comparable parameter estimates.

We used absolute concentrations of the AChE-I in our statistical models. As previous studies used dose and/or weight adapted medication concentrations ${ }^{12,14,20,29}$ we did a post hoc analysis between gene dose and dose-andweight adapted donepezil serum concentration. The effect of the gene dose was comparable to the original model although the coefficient no longer reached statistical significance. 



Figure 2 Linear regression analysis predicting donepezil serum concentration: Scatter plots, lines of fit and $95 \%$ confidence intervals for the respective independent variables.

Notes: $y$-axis: standardized serum concentration in nanogram/milliliter, $x$-axis standardized value of respective independent variables. 


\section{Factors Associated with Rivastigmine Levels}

Rivastigmine concentrations were not significantly affected by any of the variables (total daily dose, concomitant therapy with CYP2D6 inhibitors, age, sex, duration of AChE-I therapy, body weight or BMI (Supplementary Tables 13-14)). In accordance with the drug label information, we had expected to see an association between weight and rivastigmine concentration. ${ }^{15}$ Despite pointing in the expected direction, the $\beta$-coefficient for weight did not attain statistical significance, maybe due to the small sample size. There are very few other studies investigating rivastigmine plasma concentration and none of them investigated further variables that might affect rivastigmine concentrations. ${ }^{6,7,13}$

\section{Therapeutic Range of Blood Drug Concentrations}

For donepezil, the AGNP Consensus Guidelines for Therapeutic Drug Monitoring in Psychiatry recommend a therapeutic range of $50-75 \mathrm{ng} / \mathrm{mL}$ at trough steadystate. ${ }^{40}$ Following this recommendation, $73 \%$ of our subjects in the $10 \mathrm{mg}$ donepezil cohort had a drug concentration below and 5\% above this range (Figure 1). None of the subjects with a drug concentration below the recommended range was a rapid or ultra-rapid metabolizer. One of the subjects with a drug concentration above the recommended range was under medication with citalopram, a weak CYP2D6 inhibitor that usually does not cause clinically significant interactions. Having a substantial percentage of patients with a serum concentration below the recommended range has previously been described by Koeber et al, ${ }^{41}$ who found serum concentrations below $30 \mathrm{ng} / \mathrm{mL}$ in $34.5 \%$ of patients under therapy with donepezil. The authors pointed out that in some cases therapeutic concentrations of donepezil could possibly only be achieved with a dosage higher than $10 \mathrm{mg} /$ day. That we found an even higher percentage of subjects with serum concentrations below the recommended range can be explained by the increase of the lower limit from $30 \mathrm{ng} / \mathrm{mL}$ to $50 \mathrm{ng} / \mathrm{mL}$ in $2018 .^{40}$

The recommended therapeutic range for rivastigmine is between 5 and $13 \mathrm{ng} / \mathrm{mL}$ measured during trough steadystate. ${ }^{40} 65 \%$ of the participants in this study with a daily dose of $9.5 \mathrm{mg}$ rivastigmine were below and $15 \%$ above this target range (Figure 1). Therefore, 4 out of 5 subjects under therapy with rivastigmine had drug concentrations outside the recommended range, possibly resulting in less treatment efficacy or increased risk of side effects. That we had an unexpected large number of participants with rivastigmine serum concentrations below the recommended range might be due to several factors. Firstly, according to the drug label information ${ }^{15}$ mean trough rivastigmine plasma concentrations measured for the $9.5 \mathrm{mg} / 24$ hours patch are below $4 \mathrm{ng} / \mathrm{mL}$, which already is below the recommended range of 5-15 ng/mL. Secondly, the recommended range for transdermal application does not differentiate between different doses of the patch, eg, 4.6, 9.5 and $13.3 \mathrm{mg} / 24$ hours. Given that in a study by Lefevre et $\mathrm{al}^{42}$ the average maximal plasma concentration of rivastigmine under treatment with $9.5 \mathrm{mg} / 24$ hours was 7.29 $\mathrm{ng} / \mathrm{mL}$ and with $13.3 \mathrm{mg} / 24$ hours was $12.9 \mathrm{ng} / \mathrm{mL}$ raises concerns about the validity of the recommended trough concentration of $5-15 \mathrm{ng} / \mathrm{mL}$. Thirdly, considering the short half-life time of rivastigmine of approximately 3.4 hours, ${ }^{15}$ the fact that in some subjects blood draw occurred over 24 hours after the patch was applied might also have contributed to the finding. Consistent with this view, only a smaller proportion of subjects treated with donepezil with 70 hours half-life time ${ }^{25}$ were below the recommended therapeutic range.

Currently, there is a lot of debate about specifications of the therapeutic range for AChE-inhibitors. Nevertheless, looking at the blood concentration of AChEinhibitors in the case of insufficient clinical response or side effects would be recommended before discontinuing or switching medication.

\section{Correlation Between Changes in Cognitive Function Level and Drug Dose Level}

Previous studies on the correlation between cognitive functions and the blood drug concentration of either donepezil or rivastigmine showed inconsistent results. While some authors found no correlation between donepezil or rivastigmine concentrations and cognitive changes, ${ }^{13,14}$ others could demonstrate a significant association between serum concentrations and cognitive performance. ${ }^{6-9,12,28}$ In both treatment groups, we found improvements in some tests and deterioration in others at follow-up. This could be due to substantial variability in the course of the disease in individual subjects as well as varying individual responses to AChE-I treatment. Due to the progressive nature of $\mathrm{AD}$, it is difficult to estimate drug efficacy for 
single individuals as even in the event of deterioration the drug could still be effective. ${ }^{2}$

Changes in the wordlist delayed recall subtest were significantly influenced by the serum concentration of rivastigmine. Overall regression analyses of the $\beta$ coefficients of the rivastigmine serum concentration were higher than regression analyses of the $\beta$-coefficients of the donepezil serum concentration, hinting at a closer correlation between rivastigmine serum concentrations and clinical efficacy. Bullock et al demonstrated greater treatment response to rivastigmine compared to donepezil in patients younger than 75 years of age $(p<0.05) .{ }^{27}$ They hypothesized that this finding might be due to the additional inhibition of butyrylcholinesterase by rivastigmine. Blood drug concentrations were not measured in that study.

In addition, correlations between the clinical efficacy of donepezil and gene dose or metabolic profile without considering the mediating effect of serum levels could have been assessed. Yet, serum concentration is also influenced by factors other than the gene dose, such as age, sex, weight, daily dose, or duration of treatment, that was actually the case in our study (compare Table 4, Supplementary Table 9). However, we indirectly demonstrated the influence of gene dose or metabolic profile on the efficacy of donepezil by investigating the relationship between donepezil serum concentration and gene dose/ metabolic profile as well as between donepezil serum concentration and clinical efficacy.

\section{Limitations}

The recruitment was slower than expected and we were not able to recruit the aspired sample size before funds for the study ran out. Despite the fact that our sample size was smaller than expected, we were able to show significant correlations between gene dose or type of metabolizer and donepezil serum concentration.

Due to the small sample size, weak correlations might not have been detected. However, even in our small sample, the correlation between rivastigmine serum concentration and cognitive changes, measured by the wordlist delayed recall subtest, as well as the influence of the gene dose or metabolism type on the level of donepezil serum concentrations, were statistically significant.

The compliance of patients regarding their regular intake of the medication as prescribed could not be verified. All data concerning time and regularity of intake are based on patient and caregiver statements.
Patients were routinely supposed to make follow-up appointments 3-6 months after the initial diagnosis. In reality, however, there was much variability regarding time to follow-up. As, according to our results, donepezil concentration seems to depend on the duration of treatment, less variation would have been preferable.

Although we aimed to measure medication levels in the trough state as closely as possible prior to the next scheduled drug intake, for organizational reasons this was not always possible. If the blood draw did not take place before the scheduled drug administration, the AChE-I was not administered until after the blood was drawn, ie, up to a maximum of 4 hours later. On average blood was drawn $1.25 \pm 2.4$ hours before the time point of the next scheduled medication administration. Depending on the CYP2D6 genotype, a gene dose and metabolism type were allocated to each participant. Genotyping was performed on an assortment of CYP2D6 alleles that predict with almost $100 \%$ accuracy most patients without enzyme activity (PM) ${ }^{43}$ Individuals with a duplication of a working allele were categorized as UM. However, using this method only $10-30 \%$ of subjects with the phenotype UM can be detected. ${ }^{17,44,45}$ In addition, further genetic alterations explaining UM phenotype in subjects without allele duplication are as yet unknown. ${ }^{46}$ Hence, further unknown mutations of CYP2D6 gene influencing metabolism cannot be ruled out.

Another point that needs to be considered is that the cognitive functions of patients with Alzheimer's dementia are subject to fluctuation from day to day and display only one point in time. They also depend on the motivation of the patients tested. CERAD-NAB is a very wellvalidated and standardized neuropsychological assessment tool. However, inter-rater variability cannot be ruled out in our study. Also, the CERAD-NAB does not allow for any objective evidence regarding efficacy on activities of daily living or neuropsychiatric symptoms which might have improved even if CERAD-NAB scores did not.

\section{Conclusion}

As in both the donepezil and rivastigmine cohort subjects had serum drug concentrations beneath or above the respective recommended range, testing drug blood concentrations is recommended. This might be especially important for subjects treated with donepezil not responding to treatment or suffering from side effects. These subjects might further profit from additional testing of CYP2D6 polymorphisms as genetic variability of $C Y P 2 D 6$ contributes significantly to 
serum donepezil concentration. Evaluating both, serum concentration and CYP2D6 polymorphisms, might help to attribute the lack of clinical response or side effects to either adherence or genetic causes. Thereby giving the treating physician valuable information when deciding if donepezil should be replaced by another AChE-I or if a home nursing service should be involved to ensure medication is administered as prescribed.

\section{Acknowledgments}

We would like to give special thanks to the laboratory technicians Christine Uhlich and Christine Grubmüller for their continuous support and to Kate Eden and Karl Hughes from the Language Center of the Technical University of Munich for their help editing the manuscript. CYP2D6 genotyping was supported by an unrestricted research grant by Novartis. Novartis was neither involved in data collection, measurements or statistical analyses nor in the interpretation of the data.

\section{Disclosure}

Outside the submitted work, Dr Grimmer reports having received consulting fees from Actelion, Biogen, Eli Lilly, Iqvia/Quintiles; MSD; Novartis, Quintiles, Roche Pharma, lecture fees from Biogen, Lilly, Parexel, Roche Pharma, and grants to his institution from Actelion and PreDemTech. Outside the submitted work, Dr DiehlSchmid reports having received lecture fees from Novartis. Outside the submitted work, Prof. Steimer reports having received consulting fees, support or lecture fees from Roche Diagnostics, Abbott, Siemens, Microgenics, Thermo Fisher. Dr Katharina Buerger is the advisory board member for Biogen GmbH Germany, and acts as an advisor to Ernst\&Young Consulting, during the conduct of the study. Miss Marion Stange reports grants from Novartis, during the conduct of the study. The authors report no other conflicts of interest in this work.

\section{References}

1. NICE. Dementia: A NICE-SCIE Guideline on Supporting People with Dementia and Their Carers in Health and Social Care. Leicester, London: British Psychological Society; Royal College of Psychiatrists; 2007.

2. Deuschl G, Wea M. S3-Leitlinie Demenzen; 2016. Available from: www.dgn.org/leitlinien. Accessed September 12, 2017.

3. Gauthier S, Patterson C, Chertkow H, et al. 4th Canadian Consensus Conference on the Diagnosis and Treatment of Dementia. Can J Neurol Sci. 2012;39(6 Suppl 5):S1-S8. doi:10.1017/s031716 7100015183
4. Guideline Adaption Committee. Clinical Practice Guidelines and Principles of Care for People with Dementia. Sydney; 2016.

5. Doody RS, Stevens JC, Beck C, et al. Practice parameter: management of dementia (an evidence-based review). Report of the Quality Standards Subcommittee of the American Academy of Neurology. Neurology. 2001;56(9):1154-1166. doi:10.1212/ WNL.56.9.1154

6. Chou M-C, Chen C-H, Liu C-K, Chen S-H, Wu S-J, Yang Y-H. Concentrations of rivastigmine and NAP 226-90 and the cognitive response in Taiwanese Alzheimer's disease patients. $J$ Alzheimers Dis. 2012;31(4):857-864. doi:10.3233/JAD-2012-120109

7. Chen T-H, Chou M-C, Lai C-L, Wu S-J, Hsu C-L, Yang Y-H. Factors affecting therapeutic response to rivastigmine in Alzheimer's disease patients in Taiwan. Kaohsiung J Med Sci. 2017;33(6):277-283. doi:10.1016/j.kjms.2017.04.006

8. Rogers SL, Doody RS, Mohs RC, Friedhoff LT. Donepezil improves cognition and global function in Alzheimer disease. A 15-week, double-blind, placebo-controlled study. Donepezil Study Group. Arch Intern Med. 1998a;158(9):1021-1031. doi:10.1001/ archinte.158.9.1021

9. Yang Y-H, Chen C-H, Chou M-C, Li C-H, Liu C-K, Chen S-H. Concentration of donepezil to the cognitive response in Alzheimer disease. J Clin Psychopharmacol. 2013;33(3):351-355. doi:10.1097/ JCP.0b013e31828b5087

10. Lu J, Wan L, Zhong Y, et al. Stereoselective metabolism of donepezil and steady-state plasma concentrations of S-donepezil based on CYP2D6 polymorphisms in the therapeutic responses of Han Chinese patients with Alzheimer's disease. J Pharmacol Sci. 2015;129(3):188-195. doi:10.1016/j.jphs.2015.10.010

11. Miranda LFJR, Gomes KB, Tito PAL, et al. Clinical response to donepezil in mild and moderate dementia. relationship to drug plasma concentration and CYP2D6 and APOE genetic polymorphisms. $J$ Alzheimers Dis. 2017;55(2):539-549. doi:10.3233/JAD-160164

12. Hefner G, Brueckner A, Hiemke C, Fellgiebel A. Therapeutic drug monitoring for patients with Alzheimer Dementia to improve treatment with donepezil. Ther Drug Monit. 2015;37(3):353-361. doi:10.1097/FTD.0000000000000152

13. Gobburu JV, Tammara V, Lesko L, et al. Pharmacokineticpharmacodynamic modeling of rivastigmine, a cholinesterase inhibitor, in patients with Alzheimer's disease. J Clin Pharmacol. 2001;41 (10):1082-1090. doi:10.1177/00912700122012689

14. Varsaldi F, Miglio G, Scordo MG, et al. Impact of the CYP2D6 polymorphism on steady-state plasma concentrations and clinical outcome of donepezil in Alzheimer's disease patients. Eur J Clin Pharmacol. 2006;62(9):721-726. doi:10.1007/s00228-006-0168-1

15. FDA. NDA 22-083/S016 Exelon ${ }^{\circledR}$ Patch (rivastigmine transdermal system). Available from: https://www.accessdata.fda.gov/drugsatfda docs/nda/2012/022083orig1s016.pdf. Accessed August 20, 2017.

16. Meyer UA, Zanger UM. Molecular mechanisms of genetic polymorphisms of drug metabolism. Annu Rev Pharmacol Toxicol. 1997;37:269-296. doi:10.1146/annurev.pharmtox.37.1.269

17. Sachse C, Brockmöller J, Bauer S, Roots I. Cytochrome P450 2D6 variants in a Caucasian population. Allele frequencies and phenotypic consequences. Am J Hum Genet. 1997;60(2):284-295.

18. Raimundo $\mathrm{S}$, Toscano $\mathrm{C}$, Klein $\mathrm{K}$, et al. A novel intronic mutation, 2988GA, with high predictivity for impaired function of cytochrome P450 2D6 in white subjects. Clin Pharmacol Ther. 2004;76 (2):128-138. doi:10.1016/j.clpt.2004.04.009

19. Hicks JK, Swen JJ, Thorn CF, et al. Clinical pharmacogenetics implementation consortium guideline for CYP2D6 and CYP2C19 genotypes and dosing of tricyclic antidepressants. Clin Pharmacol Ther. 2013;93(5):402-408. doi:10.1038/clpt.2013.2

20. Noetzli M, Guidi M, Ebbing K, et al. Population pharmacokinetic approach to evaluate the effect of CYP2D6, CYP3A, ABCB1, POR and NR1I2 genotypes on donepezil clearance. Br J Clin Pharmacol. 2014;78(1):135-144. doi:10.1111/bcp.12325 
21. Magliulo L, Dahl M-L, Lombardi G, et al. Do CYP3A and ABCB1 genotypes influence the plasma concentration and clinical outcome of donepezil treatment? Eur J Clin Pharmacol. 2011;67(1):47-54. doi: $10.1007 / \mathrm{s} 00228-010-0883-5$

22. Cacabelos R. Donepezil in Alzheimer's disease. From conventional trials to pharmacogenetics. Neuropsychiatr Dis Treat. 2007;3 (3):303-333.

23. Steimer W, Zöpf K, von Amelunxen S, et al. Allele-specific change of concentration and functional gene dose for the prediction of steady-state serum concentrations of amitriptyline and nortriptyline in CYP2C19 and CYP2D6 extensive and intermediate metabolizers. Clin Chem. 2004;50 (9):1623-1633. doi:10.1373/clinchem.2003.030825

24. Gonzalez FJ, Skoda RC, Kimura S, et al. Characterization of the common genetic defect in humans deficient in debrisoquine metabolism. Nature. 1988;331(6155):442-446. doi:10.1038/331442a0

25. FDA. Aricept label information. Available from: https://www.accessdata.

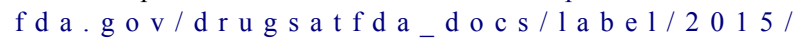
020690s039,021720s012,022568s008lbl.pdf. Accessed August 21, 2017.

26. Mori E, Ikeda M, Nakai K, Miyagishi H, Nakagawa M, Kosaka K. Increased plasma donepezil concentration improves cognitive function in patients with dementia with Lewy bodies. An exploratory pharmacokinetic/pharmacodynamic analysis in a Phase 3 randomized controlled trial. J Neurol Sci. 2016;366:184-190. doi:10.1016/j. jns.2016.05.001

27. Bullock R, Bergman H, Touchon J, et al. Effect of age on response to rivastigmine or donepezil in patients with Alzheimer's disease. Curr Med Res Opin. 2006;22(3):483-494. doi:10.1185/030079906X89685

28. Coin A, Pamio MV, Alexopoulos C, et al. Donepezil plasma concentrations, CYP2D6 and CYP3A4 phenotypes, and cognitive outcome in Alzheimer's disease. Eur J Clin Pharmacol. 2016;72(6):711-717. doi:10.1007/s00228-016-2033-1

29. Sonali N, Tripathi M, Sagar R, Velpandian T, Subbiah V. Impact of CYP2D6 and CYP3A4 genetic polymorphism on combined cholinesterase inhibitors and memantine treatment in mild to moderate Alzheimer's disease. Dement Geriatr Cogn Disord. 2014;37(1-2):58-70. doi:10.1159/000350050

30. Seripa D, Bizzarro A, Pilotto A, et al. Role of cytochrome P4502D6 functional polymorphisms in the efficacy of donepezil in patients with Alzheimer's disease. Pharmacogenet Genomics. 2011;21 (4):225-230. doi:10.1097/FPC.0b013e32833f984c

31. Ortner M, Stange M, Schneider H, et al. Serum concentrations of cholinesterase inhibitors in patients with Alzheimer's dementia are frequently below the recommended levels. Front Pharmacol. 2020;11:691. doi:10.3389/fphar.2020.00691

32. Ortner M, Kurz A, Alexopoulos P, et al. Small vessel disease, but neither amyloid load nor metabolic deficit, is dependent on age at onset in Alzheimer's disease. Biol Psychiatry. 2015;77(8):704-710. doi:10.1016/j.biopsych.2014.01.019

33. Folstein MF, Folstein SE, McHugh PR. "Mini-mental state". A practical method for grading the cognitive state of patients for the clinician. J Psychiatr Res. 1975;12(3):189-198. doi:10.1016/ 0022-3956(75)90026-6
34. Morris JC, Heyman A, Mohs RC, et al. The Consortium to Establish a Registry for Alzheimer's Disease (CERAD). Part I. Clinical and neuropsychological assessment of Alzheimer's disease. Neurology. 1989;39(9):1159-1165. doi:10.1212/WNL.39.9.1159

35. Watson YI, Arfken CL, Birge SJ. Clock completion. An objective screening test for dementia. $J$ Am Geriatr Soc. 1993;41 (11):1235-1240. doi:10.1111/j.1532-5415.1993.tb07308.x

36. McKhann GM, Knopman DS, Chertkow H, et al. The diagnosis of dementia due to Alzheimer's disease. Recommendations from the National Institute on Aging-Alzheimer's Association workgroups on diagnostic guidelines for Alzheimer's disease. Alzheimers Dement. 2011;7(3):263-269. doi:10.1016/j.jalz.2011.03.005

37. Welsh K, Butters N, Hughes J, Mohs R, Heyman A. Detection of abnormal memory decline in mild cases of Alzheimer's disease using CERAD neuropsychological measures. Arch Neurol. 1991;48 (3):278-281. doi:10.1001/archneur.1991.00530150046016

38. Lu Y, Qin X, Li S, et al. Quantitative assessment of CYP2D6 polymorphisms and risk of Alzheimer's disease: a meta-analysis. J Neurol Sci. 2014;343(1-2):15-22. doi:10.1016/j.jns.2014.05.033

39. Rogers SL, Friedhoff LT. Pharmacokinetic and pharmacodynamic profile of donepezil $\mathrm{HCl}$ following single oral doses. $\mathrm{Br} J$ Clin Pharmacol. 1998;46(Suppl 1):1-6. doi:10.1046/j.13652125.1998.0460s 1001.x

40. Hiemke C, Bergemann N, Clement HW, et al. Consensus guidelines for therapeutic drug monitoring in neuropsychopharmacology: update 2017. Pharmacopsychiatry. 2018;51(1-02):9-62. doi:10.1055/ s-0043-116492

41. Koeber R, Kluenemann -H-H, Waimer R, et al. Implementation of a cost-effective HPLC/UV-approach for medical routine quantification of donepezil in human serum. J Chromatogr B Analyt Technol Biomed Life Sci. 2012;881-882:1-11. doi:10.1016/j.jchromb.2011. 10.027

42. Lefèvre G, Sedek G, Jhee SS, et al. Pharmacokinetics and pharmacodynamics of the novel daily rivastigmine transdermal patch compared with twice-daily capsules in Alzheimer's disease patients. Clin Pharmacol Ther. 2008;83(1):106-114. doi:10.1038/sj.clpt.6100242

43. Chou W-H, Yan F-X, Robbins-Weilert DK, et al. Comparison of two CYP2D6 genotyping methods and assessment of genotype-phenotype relationships. Clin Chem. 2003;49(4):542-551. doi:10.1373/49.4.542

44. Griese EU, Zanger UM, Brudermanns U, et al. Assessment of the predictive power of genotypes for the in-vivo catalytic function of CYP2D6 in a German population. Pharmacogenetics. 1998;8 (1):15-26. doi:10.1097/00008571-199802000-00003

45. Dahl ML, Johansson I, Bertilsson L, Ingelman-Sundberg M, Sjöqvist F. Ultrarapid hydroxylation of debrisoquine in a Swedish population. Analysis of the molecular genetic basis. J Pharmacol Exp Ther. 1995;274(1):516-520.

46. Zanger UM, Fischer J, Raimundo S, et al. Comprehensive analysis of the genetic factors determining expression and function of hepatic CYP2D6. Pharmacogenetics. 2001;11(7):573-585. doi:10.1097/ 00008571-200110000-00004

\section{Publish your work in this journal}

Drug Design, Development and Therapy is an international, peerreviewed open-access journal that spans the spectrum of drug design and development through to clinical applications. Clinical outcomes, patient safety, and programs for the development and effective, safe, and sustained use of medicines are a feature of the journal, which has also been accepted for indexing on PubMed Central. The manuscript management system is completely online and includes a very quick and fair peer-review system, which is all easy to use. Visit http://www. dovepress.com/testimonials.php to read real quotes from published authors. 